Mutations in $B M P R 2$ are the major cause of familial pulmonary arterial hypertension (PAH). Reduced BMPR-II expression is significantly reduced in both familial and idiopathic PAH patients. We have shown previously that BMPR-II expression is regulated via a lysosomal degradative pathway. The anti-malarial drug, chloroquine, blocks lysosomal degradation by raising lysosomal $\mathrm{pH}$ and impairs autophagic protein degradation. Using an experimental rat model of $\mathrm{PAH}$ we observed that chloroquine administration prevented an increase in right ventricular systolic pressure (RVSP), right ventricular hypertrophy (RVH) and vascular remodelling following monocrotaline (MCT) treatment. BMPR-II expression was significantly increased in lungs from chloroquine treated rats. Furthermore, in cellular localisation studies chloroquine increased BMPR-II cell surface expression.

Methods Male Sprague-Dawley rats received a single subcutaneous injection of MCT to induce PAH. To assess prevention or inhibition of $\mathrm{PAH}$ progression, animals received chloroquine, or vehicle, by daily intraperitoneal injection from day 1 to 21 or day 21 to 31 post MCT injections, respectively. Rats were anaesthetised for haemodynamic assessment and lung tissue collected for immunohistochemistry and protein isolation. Muscularisation of small pulmonary arteries was assessed in lung tissue sections by staining with anti-smooth muscle actin. BMPR-II protein expression was determined in frozen lung tissue using western blotting. Cellular localisation of BMPR-II expression in a lung fibroblast cell line that stably expresses green fluorescent protein (GFP) tagged BMPR-II was determined using immunofluorescence and biotinylation of cell surface BMPR-II using NHS-Biotin-SS labelling and precipitation with avidin agarose beads. BMPR-II cell surface expression was then determined by GFP immunoblotting.

Results Chloroquine prevented experimental PAH by significantly decreasing RVSP, RVH and muscularisation in MCT-treated rats. Treatment with chloroquine dramatically increased BMPR-II protein levels in the lung. Furthermore, chloroquine treatment inhibited MCT-induced PAH. This was associated with an increase in cell surface BMPR-II expression in a cell line that stably expresses the GFP-tagged receptor.

Conclusion This study demonstrates the potential use of chloroquine as a therapeutic agent in the treatment of PAH by potentially increasing levels of BMPR-II at the cell surface.

\section{S100 THE BONE MORPHOGENETIC PROTEIN TYPE II RECEPTOR IS CRITICAL FOR VENOUS ANGIOGENESIS IN ZEBRAFISH}

doi:10.1136/thoraxjnl-2011-201054b.100

${ }^{1} \mathrm{~J}$ E Cannon, ${ }^{2} \mathrm{C}$ R Bradshaw, ${ }^{3} \mathrm{~J} \mathrm{C}$ Smith, ${ }^{4} \mathrm{~N}$ W Morrell. ${ }^{1}$ Gurdon Institute and Departments of Medicine and Zoology, University of Cambridge, Cambridge, UK; ${ }^{2}$ Gurdon Institute, University of Cambridge, Cambridge, UK; ${ }^{3}$ MRC National Institute for Medical Research, London, UK; ${ }^{4}$ Department of Medicine, University of Cambridge, Cambridge, UK

Introduction and Objectives Pulmonary arterial hypertension (PAH) is a rare but severe condition, often fatal within 3-5 years due to right heart failure. Mutations in the bone morphogenetic protein (BMP) type II receptor (BMPR-II) underlie heritable forms of the disease but the mechanisms leading to vascular disease remain obscure from studies in mice and humans. Here we use zebrafish, which have a well-documented pattern of angiogenesis, as a model organism to address this question.

Methods The transgenic $\operatorname{Tg}(f l i 1 a: e g f p)$ zebrafish line, where the fli1a promoter drives GFP expression in vascular endothelial and blood cells, was used throughout this work. A variety of methods were used to dissect the role of BMP signalling in vascular development including: (i) BMP receptor inhibitors (dorsomorphin and LDN193189), (ii) antisense morpholino oligonucleotides (morpholinos) and (iii) transgenic zebrafish engineered with heat shock inducible dominant-negative BMP receptors. To identify BMP responsive transcripts from vascular endothelial cells in vivo we developed a system to allow FACS isolation of GFP+ve cells dissociated from $\operatorname{Tg}($ flila:egfp) zebrafish following incubation in LDN193189 or DMSO solvent control. The mRNA transcripts in GFP+ve cells were determined using massively parallel (Illumina) sequencing, mapped to the Zv8 zebrafish genome and differences in transcript abundance between LDN193189 and DMSO treated embryos were determined using cufflinks.

Results Inhibition of BMP signalling with LDN193189 in zebrafish embryos after dorso-ventral patterning has occurred blocked venous but not arterial angiogenesis. This phenotype was reproduced in $B M P R 2$ dominant-negative zebrafish and following knockdown of the zebrafish homologues of BMPR2, bmpr2a and bmpr2b with morpholinos. Illumina sequencing identified the BMP responsive genes in vascular endothelial cells and the gene(s) responsible for the venous angiogenic phenotype are being determined by knockdown of these genes with morpholinos.

Conclusion BMP signalling, via $B M P R 2$, is critical for venous but not arterial angiogenesis in zebrafish. BMPR2 mutations are recognised as causative in patients with heritable $\mathrm{PAH}$, where venous involvement is recognised, but also in pulmonary veno-occlusive disease. Our findings provide novel insights onto the potential role of the pulmonary venous system in pulmonary hypertension and implicate abnormal venous angiogenesis as a novel mechanism underlying this disease.

\section{S101 HETEROZYGOUS LOSS OF BMPR-II PREDISPOSES TO INFLAMMATORY CYTOKINE SECRETION AND PULMONARY VASCULAR SMOOTH MUSCLE PROLIFERATION}

doi:10.1136/thoraxjnl-2011-201054b.101

${ }^{1} \mathrm{E}$ Soon, ${ }^{1} \mathrm{~A}$ Crosby, ${ }^{2} \mathrm{M}$ Southwood, ${ }^{2} \mathrm{~J}$ Pepke-Zaba, ${ }^{1} \mathrm{P}$ Upton, ${ }^{1} \mathrm{~N}$ W Morrell. ${ }^{1}$ University of Cambridge, Cambridge, UK; ${ }^{2}$ Papworth Hospital NHS Trust, Cambridge, UK

Introduction Elevated levels of inflammatory cytokines are associated with idiopathic and heritable pulmonary hypertension (PAH) and predict mortality, but their role in the pathobiology of $\mathrm{PAH}$ and the underlying mechanisms remain unclear. We investigated whether loss of bone morphogenetic protein receptor type II (BMPR-II), the underlying cause of heritable $\mathrm{PAH}$, predisposes to increased inflammatory cytokine expression and pulmonary vascular remodelling.

Methods Regulation of cytokine expression by lipopolysaccharide (LPS) was studied in two in vitro models: (1) mouse pulmonary artery smooth muscle cells (PASMCs) heterozygous for a null allele in bmpr2 (BMPR2 \pm ), and (2) human PASMCs with a mutation in BMPR2 (BMPR2mut) and their wild-type counterparts. Cytokine production, regulation and effects on the pulmonary vascular system were examined in an in vivo model using the bmpr2 \pm mouse. Results Both mouse and human in vitro models showed that loss of $B M P R 2$ function leads to increased mRNA expression and secretion of interleukin 6 and IL- 8 both at baseline and after stimulation with LPS. This was associated with loss of expression of antioxidant enzymes such as superoxide dismutase 1 (SOD1) and SOD3, which is demonstrable in both mouse bmpr2 \pm and human BMPR2mut cells. Treatment with the superoxide dismutase-mimetic, Tempol, partially reversed the exaggerated cytokine response to LPS but did not affect the underlying baseline increase. We demonstrated increased phospho-Stat3 signalling and pro-proliferative and pro-survival effects of IL-6 in BMPR2mut PASMCs which is not seen in the wildtype. This was confirmed in the in vivo model, as bmpr2 \pm mice demonstrated increased IL- 6 and IL- 8 expression in sera, lung and liver tissue 3 and $24 \mathrm{~h}$ after exposure to $10 \mu \mathrm{g}$ of LPS. A similar pattern of antioxidant enzyme reduction was also seen in lung tissue from these LPS-treated bmpr2 \pm mice. 
Conclusions Loss of BMPR-II predisposes to both an abnormal increase in IL-6 and IL-8 after stimulation with LPS and a dysfunctional response to these cytokines in PASMCs and mice. The mechanism for this involves loss of antioxidant function. This abnormal response may underlie the additional lung-specific trigger that promotes the development of PAH in patients with BMPR-II mutations, and may represent a target for future therapeutic interventions.

\section{S102 THE CROSSTALK OF PDE INHIBITOR WITH BMP SIGNALLING PATHWAY IN HUMAN PULMONARY ARTERIAL SMOOTH MUSCLE CELLS}

doi:10.1136/thoraxjnl-2011-201054b.102

J Yang, X Li, C Wu, N Morrell. University of Cambridge, Cambridge, UK

Rational Sildenafil, a potent PDE inhibitor, is an established treatment for PAH. However, the detailed mechanism of its effects on the proliferation of human pulmonary artery smooth muscle cells (hPASMCs) remains unclear.

Objective Because sidenafil is effective treatments for clinical PAH, we hypothesised that these agents enhance Smad1/Id signalling through cGKI in hPASMCs.

Methods and Results Sildenafil alone has no effect on Smad1 phosphorylation and Id1 gene expression in hPASMCs, However in the presence of BMP4 Sidenafil indeed enhanced BMP-induced phosphorylation of Smad1/5 and Id1 expression in a cGMP/cGKIdependent manner in hPASMCs. The presumed mechanism is by elevation of intracellular cGKI activity which modulate smad1 phosphorylation and nuclear localisation. Knock down cGKI or use pharmalogical cGMP inhibitor abrogate the effect of Sildenafil on hPASMCs. Furthermore we confirm the rescued pSmad1 signal and elevated proliferation inhibitory effect in hPASMCs from familial pulmonary arterial hypertension patients by Sildenafil.

Conclusions Sildenafil enhance BMP/Smad through cGMP/cGKI pathway to modulate hPASMCs proliferation.

\section{Clinical studies in bronchiectasis S103 MICROBIAL COMMUNITY COMPOSITION IN THE LUNGS OF
PATIENTS WITH CYSTIC FIBROSIS AND NON-CF BRONCHIECTASIS}

doi:10.1136/thoraxjnl-2011-201054b.103

${ }^{1} \mathrm{G}$ G Einarsson, ${ }^{2} \mathrm{E}$ Klem, ${ }^{2} \mathrm{~A}$ A Fodor, ${ }^{1} \mathrm{~L}$ Wei, ${ }^{3} \mathrm{M}$ Drain, ${ }^{2} \mathrm{M} \mathrm{C}$ Wolfgang, ${ }^{3} \mathrm{~J} \mathrm{~S}$ Elborn, ${ }^{1} \mathrm{M}$ M Tunney. ${ }^{1} \mathrm{CF}$ and Airways Microbiology Research Group, Queen's University Belfast, Belfast, UK; ${ }^{2}$ University of North Carolina at Chapel Hill, Chapel Hill, North Carolina, USA; ${ }^{3}$ Centre for Infection and Immunity, Queen's University Belfast, Belfast, UK

Introduction and Aims Persistent bacterial infection is a major cause of morbidity and mortality in patients with both Cystic Fibrosis (CF) and non-CF Bronchiectasis (non-CFBX). Numerous studies have shown that CF and non-CFBX airways are colonised by a complex microbiota. However, many bacteria are difficult, if not impossible, to culture by conventional laboratory techniques. Therefore, molecular detection techniques offer a more comprehensive view of bacterial diversity within clinical specimens. The objective of this study was to characterise and compare bacterial diversity and relative abundance in patients with CF and non-CFBX during exacerbation and when clinically stable.

Methods Sputum samples were collected from CF ( $\mathrm{n}=50$ samples) and non-CFBX ( $\mathrm{n}=52$ samples) patients at the start and end of treatment for an infective exacerbation and when clinically stable.
Pyrosequencing was used to assess the microbial diversity and relative genera (or the closest possibly taxonomic order) abundance within the samples. Each sequence read was defined based on 3\% difference.

Results High-throughput pyrosequencing allowed a sensitive and detailed examination of microbial community composition. Rich microbial communities were apparent within both CF (171 specieslevel phylotypes per genus) and non-CFBX airways (144 species-level phylotypes per genus). Relative species distribution within those two environments was considerably different; however, relatively few genera formed a core of microorganisms, representing approximately $90 \%$ of all sequences, which dominated both environments. Relative abundance based on observed operational taxonomic units demonstrated that the most abundant bacteria in CF were Pseudomonas (28\%), Burkholderia (22\%), Streptococcus (13\%), family Pseudomonadaceae (8\%) and Prevotella (6\%). In contrast, the most commonly detected operational taxonomic units in non-CFBX were Haemophilus (22\%), Streptococcus (14\%), other (unassigned taxa) (11\%), Pseudomonas (10\%), Veillonella (7\%) and Prevotella (6\%).

Conclusions These results suggest that distinctive microbial communities are associated with infection and/or colonisation in patients with both CF and non-CFBX. Although relatively high species richness was observed within the two environments, each was dominated by different core taxa. This suggests that differences in the lung environment of these two diseases may affect adaptability of the relevant bacterial taxa.

\section{S104 THE CULTURE MICROBIOME IN THE LUNGS OF PATIENTS WITH COPD}

doi:10.1136/thoraxjnl-2011-201054b.104

${ }^{1} \mathrm{G}$ G Einarsson, ${ }^{2} \mathrm{D}$ Comer, ${ }^{1} \mathrm{M} \mathrm{M}$ Tunney, ${ }^{2} \mathrm{~J} \mathrm{~S}$ Elborn. ${ }^{1} \mathrm{CF}$ and Airways Microbiology Research Group, Queen's University Belfast, Belfast, UK; ${ }^{2}$ Centre for Infection and Immunity, Belfast, UK

Introduction and Aims Previous studies have shown that the lungs of Cystic Fibrosis (CF) and bronchiectasis (BE, not caused by CF) patients are colonised by a range of aerobic and anaerobic bacteria. As bacteria are also implicated in the pathogenesis and progression of chronic obstructive pulmonary disease (COPD), this study aimed to determine the culture microbiome of the COPD airways.

Methods Samples were collected from 13 stable COPD patients during routine bronchoscopy. Bronchial washings were taken at a single location in the right middle lobe by flushing and removing $30 \mathrm{ml}$ of sterile saline. Samples were cultured under strict anaerobic conditions with bacteria detected by plating on both selective and non-selective agar media and quantified by total viable count (TVC). Identification of the cultured bacteria was performed by amplification and subsequent sequencing of the 16sRNA gene.

Results Mean $\mathrm{FEV}_{1}$ was 1.36 (range 0.84-2.26, mean per cent predicted $\mathrm{FEV}_{1}, 54 \%$ ), and the mean ratio $\left(\mathrm{FEV}_{1} / \mathrm{FVC}\right)$ was $51 \%$ Bacteria were detected in $12 / 13$ samples (92\%) with bacteria from the genera Streptococcus [12/13 samples, 92\%; mean (range) TVC $\left.9.62 \times 10^{5} \mathrm{cfu} / \mathrm{ml}\left(1.50 \times 10^{3}-1.42 \times 10^{7}\right)\right]$ and Haemophilus [4/13 samples, $31 \%$; mean (range) $6.40 \times 10^{4} \mathrm{cfu} / \mathrm{ml}$ $\left.\left(2.20 \times 10^{3}-1.60 \times 10^{5}\right)\right]$ most frequently detected. Anaerobic bacteria primarily from the genera Prevotella [8/13 samples, $62 \%$; mean (range) TVC $\left.1.12 \times 10^{4} \mathrm{cfu} / \mathrm{ml}\left(1.30 \times 10^{3}-4.20 \times 10^{4}\right)\right]$ and Veillonella $\left[5 / 13\right.$ samples, $38 \%$; mean (range) TVC $1.29 \times 10^{5} \mathrm{cfu} / \mathrm{ml}$ $\left.\left(4.20 \times 10^{3}-3.60 \times 10^{5}\right)\right]$ were also detected. Pseudomonas and Moraxella were not detected in any samples.

Conclusions Our results show that bacteria from the genera Streptococcus, Haemophilus, Prevotella and Veillonella are frequently present the airways of patients suffering from COPD. Taking account of the 